\title{
What is the extent, range and nature of evidence available around the impact of 12-hour nursing shift patterns?
}

\author{
Ruth Harris \\ From The European Academy of Nursing Science EANS Summer Conference \\ Barcelona, Spain. 8-9 July 2015
}

\section{Background}

12-hour shift patterns in nursing are increasingly prevalent in health and care organisations. Key drivers are potential financial savings, perceived impact on staff recruitment/ retention and improved continuity of care. However, there are concerns that longer shifts may have a detrimental impact on patients, staff, service delivery/productivity, and access to healthcare staff.

\section{Methods}

A comprehensive scoping review using Arksey \& O'Malley's methodological framework [1] was undertaken in 2013-2014 to answer the question 'What is the extent, range and nature of evidence available around the impact of 12-hour nursing shift patterns?' A wide range of electronic databases were searched; papers identified were independently reviewed by 2 reviewers.

\section{Results}

158 potentially relevant papers were published between 1973 and 2014; 85 primary research studies and 10 reviews were included. These addressed 5 themes: risks to patients, patient experience, risks to staff, staff experience and impact on the organisation of work. Evidence of the effects of 12-hour shift patterns is inconclusive in all 5 themes, with some studies demonstrating positive impacts and others negative or no impacts.

\section{Implications}

There is insufficient evidence to justify widespread implementation or withdrawal of 12-hour shifts. The benefits and risks of 12-hour shifts for patients and staff

Correspondence: Ruth.Harris@sgul.kingston.ac.uk

Faculty of Health, Social Care and Education, Kingston University and St George's, University of London, UK 